Giovanni Ferrero
Department of
Mathematics
University of Parma
giovanni.ferrero@unipr.it
Celestina Cotti
Department of
Mathematics
University of Parma
celestina.cotti@fastwebnet.it
Michela Rossi
Politecnico di Milano
michela.rossi@polimi.it
Cecilia Tedeschi
Faculty of Engineering
University of Parma
ce.te@libero.it

Didactics

\title{
Geometries of Imaginary Space: Architectural Developments of the Ideas of M. C. Escher and Buckminster Fuller
}

Abstract. The aim of this paper is to show how efficient mathematical models can be in simulating situations that are apparently distant from one another. The works of M. C. Escher and Buckminster Fuller are used as starting points for the exploration of the first applications of non-Euclidean geometry to architecture. The investigation goes on to fractals and chaos, nurbs, blob architecture and deconstructivism.

Keywords: M. C. Escher, Buckminster Fuller, H.M. S. Coxeter, Benoit Mandelbrot, chaos theory, fractals, nurbs, blob architecture, deconstructivism

\section{Introduction}

A great variety of examples confirms the relationship between mathematics and architecture. The aim of this paper is to show how efficient mathematical models can be in simulating situations that are apparently distant from one another.

Some questions immediately arise concerning the teaching of architecture and mathematics in architecture schools. Which is the appropriate role of mathematics in architecture? Which is the architectural use of mathematics? "What" must students know nowadays? Mathematics is a fundamental calculation tool for the structural planner; but the mathematical foundations embedded in computer science are also important, since these govern design and representation, thanks to the ability of computers to make it possible to visualize virtual buildings.

Mathematics sometimes appears in architecture itself. The reference to geometry as a design tool is evident in many examples, such as, for instance, when a search for numerical and geometrical programs is applied to the organization of rooms in a design.

In order to answer these questions, it is necessary to estimate "how" and "if" mathematics plays an active role in the design and consequently, if it can be considered a dynamic instrument in the design process. Therefore, starting from Euclidean geometry and its derivations, it is necessary to verify the actual existence of relationships between mathematical research and architectural research in order to understand if the former has influenced the latter; and finally if progress in modern mathematics can still produce innovative formal results.

When mathematical models are constructible, they become real architecture, but even when they are not constructible, they still inspire architects. For a long time architects were satisfied with elementary shapes, and used forms and surfaces generated from the 
movement of relatively simple curves, such as ellipses and spirals. Their genius mainly lay in realizing buildings of great complexity and fascination based on very simple models. But today this simplicity seems to have lost its power to stimulate.

If mathematics suggests ideas for architecture, then modern research should trigger the interest of the architects, not only because of their possible applications in computer graphics - which remains a mediating instrument - but mostly because of the possibility to develop innovative building shapes.

How did the "other" geometries, posterior to Euclidean geometry, influence architecture? Are the new modern mathematical studies destined to leave a sign in modern buildings? Can architecture be inspired by non-Euclidean geometry, in its material being, which remains very distant from the virtual one?

In some recent buildings, computer-aided design makes use of curved surfaces having particular properties, such as nurbs, or it develops shapes from fractal models, which take advantage of the random repetition of simple algorithms. These phenomena may take place without the architect being directly aware of the mathematical science behind it.

Thus there is a close relationship between form and formula.

The central question is always the actual materiality of architecture, which needs to be effectively built and which is linked to the reality of three-dimensional Cartesian space. The fourth dimension of "time" appears in the rhythmic partitions that link architecture to music, but it remains rather marginal, because architecture is generally meant to be "immovable" and "eternal". It is even more difficult to design buildings in a $n$-dimensional space, as those suggested by some post-Euclidean geometries.

Mathematicians, whose constructions rely on logic and not on statics, have a greater degree of freedom than architects. But while they can freely follow their imagination, they sometimes need the help of drawings in order to explain their forms. In fact, drawing plays a determining role in the relation between geometry and the art of building, thanks to its ability to reduce the three-dimensional essence of the architectural space to the twodimensional sheet of paper. This emphasizes the dichotomy between the idea and its representation, thus between imagination and image. However, drawing is sometimes also able to illustrate the underlying mental space.

Few mathematicians and architects interact beyond expressing a mutual but generic interest for their different skills. In contrast, we chose to organize this research as a common work, confronting diverse disciplinary specializations and skills, and occasional misunderstandings of technical language. The first step was the preliminary collection of meaningful works of architecture, and their evaluation on a mathematical basis. The goal of the research is to investigate the possibility of creating new shapes from modern mathematical concepts.

\section{Escher's works}

The works of some artists generically related to Expressionism, such as M. C. Escher (1898-1972) [Escher 1982] and Buckminster Fuller (1895-1983) [Gorman 2005], are an interesting starting point for the exploration of the first applications of non-Euclidean geometry to architecture. Starting in the first decades of the twentieth century, both Escher

306 G. FERRERO, C. COTTI, M. Rossi, C. TEDESCHI - Geometries of Imaginary Space 
and Fuller based their studies on mathematics, and thanks to their acquaintance with some mathematicians, their creations anticipated some contemporary architectural research.

The lifelong research of M.C. Escher inquires into the "geometrical structures" of space, producing complex constructions that stress the ambiguities between the drawing and the projective space. His work starts from the study of regular grids, as a main reference and measuring tool of infinite space. Although Escher was not an architect in the strict sense of the word, but rather a graphic artist, he gave a visible image to the relationships between mathematics and architecture. Through geometry, his drawings investigate mental constructions and imaginary worlds, raising tricky questions about the structure of physical reality and its sensorial perception. These graphic constructions transport us to a world that seems to be physically impossible, but which is as logical as the laws of mathematics.

Speaking about his own work, Escher said that it was beautiful and ugly at the same time: beautiful, because the drawings, which are the result of research that goes far beyond its first achievements, are astonishing; ugly, because it remains unresolved, lying in the ambiguous realm between physical space and perception, in an obsessive game between the two dimensions of the drawing, the three dimensions of the Euclidean space, and the many of the sensorial space-time. "I have played a lot of tricks, and I have had a fine old time expressing concepts in visual terms, with no other aim than to find way of putting them onto paper. All I am doing in my prints is to offer a report of my discoveries" [Ernst 1995: $14]$.

Escher's studies of symmetry recall Semper's theories on the genesis of architectural and industrial design shapes [Semper 1860-63], and Gombricht's analyses on the concept of order [Gombricht 1979]. Escher searched for mathematical perfection in a chaotic universe; in his acceptance speech for the 1964 Hilversum prize, he testified "that we live in a beautiful and ordered world and not in a chaos, without rules, as it can seem" [Escher 1982: 124].

Escher thought that the laws of mathematics and geometry are not constructions of the human mind, but "they are", as the crystals "exist" in their perfect shapes. Therefore, the beauty, order and symmetry of Plato's regular solids, together with the creations of nature, become unavoidable models.

His whole work illustrates an imaginary world, which is built up on the logical bases of geometry, drawn both in plan and in space, thanks to the repetitive use of grids and symmetry. He gives a "visible" shape to some abstract concepts suggested by mathematics, such as the "infinite", the dichotomy between unity and continuity of the numerical sequence, and space-time relativity. He emphasizes the paradoxes of perception in representation, playing with the ambiguity of the projective systems. Thus, Escher illustrates his world thanks to the regular structures of the drawing; his study is based on Euclidean geometry, but he does not hesitate to follow other paths to express the paradoxes of imagination. He creates a visualization of abstract concepts coming from the studies of the mathematicians and the crystallographers, with whom he establishes a fortunate collaboration. The use of modular grids completes his systematically regular division of the plan and extends to the three-dimensional symmetries in the space. The symmetries of regular polyhedra had already triggered Plato's and Leonardo's interest before him, but Escher goes beyond the initial postulates, and pushes the formal logic to its extreme consequences. In a consistent manner, Escher follows the road that the mathematicians 
show him, going beyond the familiar world of Euclidean geometry and codified drawing techniques.

The leitmotiv of those graphical constructions of imaginary spaces is the striving to actually draw the infinite. This topic comprises both the concepts of unity and variety. So its representation becomes a cosmogony, giving an image of the created one: "We find it impossible to imagine that somewhere beyond the furthest stars of the night sky that there should come an end to space, a frontier beyond which there is nothing more [Ernst 1995: $102]$.

What is larger than the infinite? Is it possible to simulate it in a model?

In past centuries, architects and artists tried to represent the infinite depth of space. The interior space of a Gothic cathedral was intended to give the feeling of an endless room. Later, Renaissance painters found a different answer to the problem thanks to perspective, which was able to simulate the infinite depth of space on the sheet of paper or canvas. Here mathematics seem to follow painting. Some time later, the study of conic sections, together with projective geometry and the codification of descriptive geometry - based on the representation of the improper point - produced the spectacular Baroque architecture. This is also the time when quadratura, illusionistic painting usually applied to ceilings, and anamorphosis appear, in which it becomes difficult to distinguish the actual space from the perceived space.

In a way that is similar to the cathedrals builders and the Renaissance painters, but with a different approach, Escher succeeds in "building the infinite" by means of the reproduction of cyclical divisions of the plan applied to the surface of the sphere. By doing so, he eliminates the actual difficulty of repeating a same pattern on a plane surface ad infinitum, and the continuity of an endless drawing is achieved on a closed, continuous surface.

The cyclical tessellation, inspired by the geometry of arabesque decoration, was the starting point of Escher's research. The regular division of the plan recalls the principles defined in the late-nineteenth-century by the grammars of ornament. In antiquity, those were also applied to the patterns used in wall painting. Escher extends the model to the pure concepts of surface and space, adapting his drawing patterns to the spherical continuous surface. Thanks to the multiple symmetries of the five regular solids, he is able to construct drawings without interruptions: the surface of each face is finite, but the whole solid surface and the variety of its symmetries is endless. It would be impossible to realise a surface of limitless extension on the plane. Circumscribing a spherical surface, the symmetries of the polygonal faces of the regular polyhedra produce spatial symmetries. However, its projection on the plane forms radial grids, like those of rose windows and floor patterns. Therefore, radial patterns can be read as projective transformations of threedimensional shapes, such as domes, emphasizing the ambiguity between design and drawing.

While perception may be an illusion, the rigorous logic of mathematics explains the geometrical structure of the space. So the image of the "infinite" appears in the continuous growth of spirals, in Moebius strips, and in the drake biting its own tail.

In Escher's creations, some contemporary studies regarding perception and the relation between figure and background are already present, visible in the regular black and white

308 G. FerRero, C. COTTI, M. ROSSI, C. TEDESCHI - Geometries of Imaginary Space 
divisions of the plane. The graphical oppositions and the contrast between opposites become a pretext for revealing the total fusion of antithetical concepts: day and night, angel and devil, but also over and under, inside and outside. These last are expressed in a sophisticated way in his perspective studies, where he shows the illusion of the perceptive space, through the logical rationality of the paradox. Contradictions in the drawing reveal the ambiguity between the feeling of the spatial structures and their infinite depth.

Escher is not satisfied by the compromise of Renaissance linear perspective, which comes from a simplified concept of vision. His ambiguous applications of perspective in the depiction of impossible architectures testify to his dissatisfaction with the rules of codified drawing. His perspective studies attempt to follow the physiology of vision, the mobility of the eye and the relativity of time to perception. His curved spaces are far from Panofsky's theories about the perspective knowledge in antiquity [Panofsky 1997], but it is quite easy to establish a link between ancient perspective and Escher's projective constructions.

In fact, Euclidean geometries were not sufficient for his research. The compositions related to Poincaré's drawing of hyperbolic space (Circle limit I, II, III, IV) show a relationship to mathematical studies about "other" geometries, and the symmetries of polytopes. Escher is perhaps the first artist to be interested in the abstract concepts of contemporary mathematics, and he is able to illustrate these concepts, pointing the way to more recent architectural research. He gives representational shape to the structures of some non-Euclidean geometries, such as in the grid deformations of Print Gallery (related to a Riehman surface with its empty centre). But he also anticipates some later concepts, like those of Mandelbrot's fractals, in Square Limit, designed in 1964.

\section{Fuller's works}

Escher's graphical inventions are reflected in the works of architect-engineer Buckminster Fuller. Fuller studied the spatial symmetries of the regular polyhedra in order to solve structural problems, and he applied this geometry to the design of light, prefabricated and transportable structures. A mathematical program, derived from the geodetic divisions of the sphere and/or the multiple symmetries of the polytopes, generated both an architectural shape and an actual construction. He then applied the same model to the problem of representing the earth, coming up with an innovative cartographic projection, especially considered for use in air navigation.

Fuller's domes offer a good field of application for testing the architectural potential of Escher's inventions and suggestions inspired by mathematicians.

H. M. S. Coxeter (1907-2003), a friend of both Escher and Fuller, is the key figure for the comprehension of the recent mathematical interpretations of architecture based on topology and chaos theory. The starting point of Coxeter's research was the symmetrical structures of the kaleidoscope, from which he developed the study of the polytopes. These are complex objects in $n$-dimensional space; they do not exist in the real world, but they can be mathematically described. They inspired Escher's work on Circle limits. ${ }^{1}$ Fuller's domes are also inspired by Coxeter's studies of space symmetry, which would lead to the discovery of a new shape of carbon crystallization. "Buckyballs" are empty spheres with 60 carbon atoms on their surface which form a grid of hexagons and pentagons, like in Fuller's geodesic domes, for which they are named. ${ }^{2}$ 


\section{Non-Euclidean geometries and architecture: meaningful examples}

Chaos theory and fractal geometry, which inspire deconstructive architecture, are in contrast with these tidy symmetries. In fact, deconstructive architecture abandons orthogonal cages, and allows the volumes to break out, creating apparently unordered organic shapes, such as those of blob architecture.
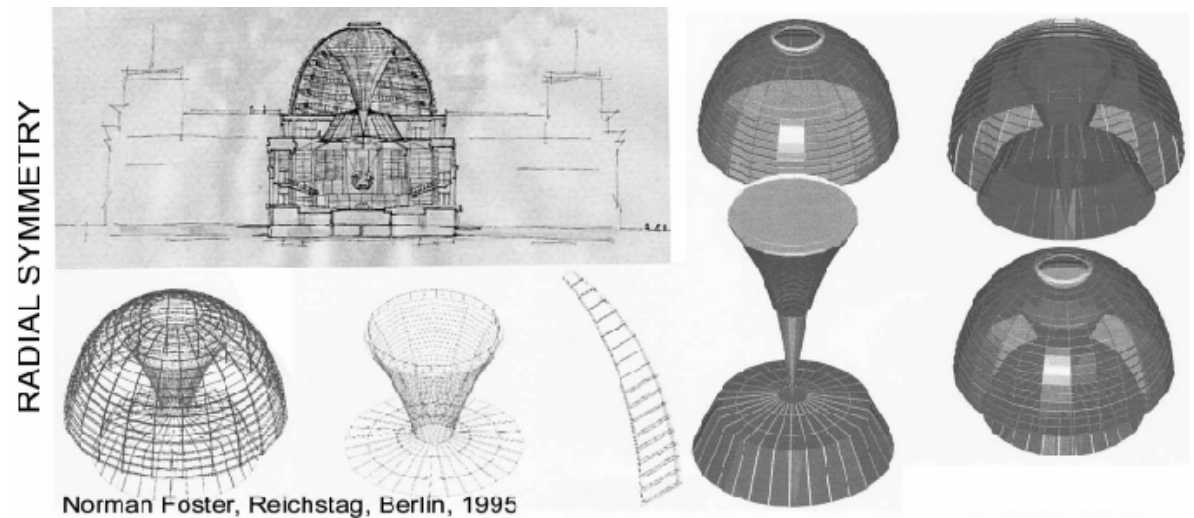

Norman Foster, Reichstag, Berlin, 1995
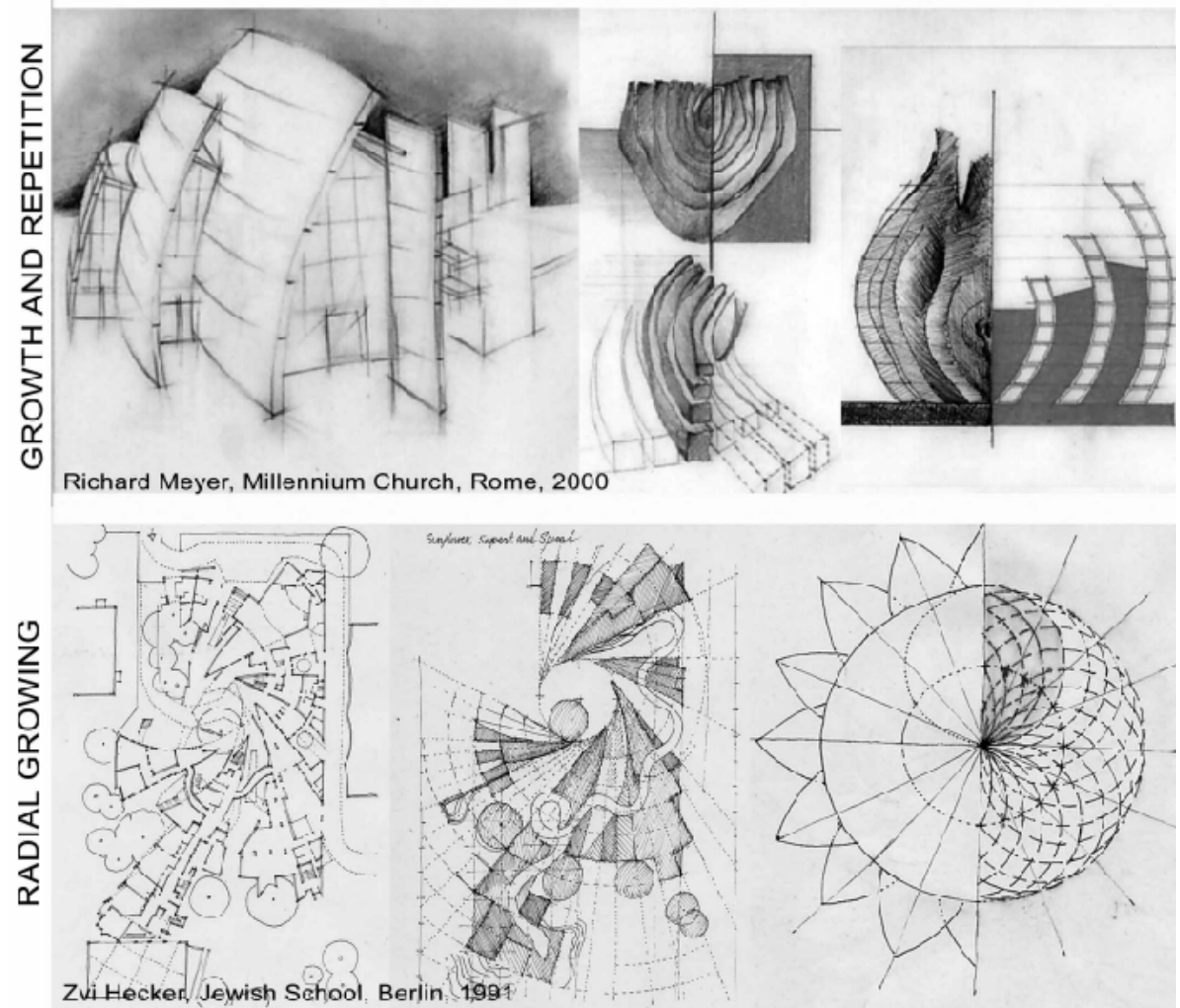

Fig. 1.

310 G. FerRero, C. COTTI, M. Rossi, C. TEDESCHI - Geometries of Imaginary Space 
Fractal geometry, defined by Polish mathematician Benoît B. Mandelbrot (1924 -) describes some objects that are generated by using repetitive algorithms, reproducing selfsimilar shapes like the branching of trees, ferns and cauliflowers. From these models both mathematicians and architects have drawn design inspiration.

But repetitive principles also appear in the symmetries of plane tessellation, or in the growing construction of the spirals. Self-similar structures can also be found in the design of the Indian kolam and in Celtic knots. In architecture, this concept appears in the spiral dome of S. Ivo alla Sapienza in Rome by Borromini (1642), in the repetition of octagonal shapes in Castel del Monte in southern Italy (mid-thirteenth century), in the elliptic domes of San Carlino alle Quattro Fontane in Rome by Borromini (1634), or in the network of arches that forms the dome of the Chapel of the Holy Shroud in Turin by Guarini (1666), among many other examples.

Finally, there is a reference to fractals in many works of modern architecture: in the selfsimilar triangles of Palmer House by Frank Lloyd Wright (1950-51) [Eaton 1998]; in the spherical shells of the Opera House in Sidney by Jørn Oberg Utzon (1957-73); in the sails of the Chiesa della Divina Misericordia by Richard Meyer in Rome (2003); and in some works by Zvi Hecker, like the Jewish school of Berlin (1991), the spiral apartment house in Ramat Gan (1981-1989), the polygons of the Hebrew University in Jerusalem (fig. 1).

The mathematical research into "chaotic order" helps us to understand the articulated shapes of "Deconstructivism", 3 including the recent Bird's Nest stadium in Beijing by Herzog and De Meuron (2008). These complex works of architecture, with surfaces that are bent or broken, achieve a controlled disorder through reiterated permutations of regular or simple shapes, which are difficult to conceive without computer elaboration.

Thanks to a controlled randomness, the computer introduces a chaotic element into the architecture layout, applying models that imitate the contemporary world, where it is difficult to recognize a classical concept of order.

Architecture evokes the continuous stress between the tidy cosmos of Creation and the chaos that preceded it, with surfaces which suggest continuous transformations. In these works of architecture, inspired by the earliest studies in topology, spaces no longer obey Cartesian rigor, but imply a continuous formal evolution. The walls are tilted and open: the concept of enclosure is broken by transformations that follow topological criteria (according to which a cube can become a sphere). Bending and distortions are achieved through a succession of shape deformations described by non-uniform rational B-spline (nurbs) surfaces. A meaningful example is the Prototype House in Long Island by Greg Lynn, composed by three altered volumes.

More recently, architectural design was inspired by the organic shapes of blob architecture, of which Greg Lynn is the main exponent. His work contrasts the natural laws of static with dynamic and interactive shapes, generated from the simulation of customers flows, like in the St. Gallen Kunstmuseum (2001). Blob architecture is the result of the application of deforming forces on initial shapes, emphasizing the elasticity of the volumes.

The role of the tool used for representation in the design process is fundamental, since the software programs commonly used in architectural design are based on geometrical and mathematical algorithms. Architects therefore strive (sometimes successfully) to give material form to the ideas of the mathematicians, thus generating new shapes. 
Escher's theory on the regular division of the plane was the first step towards broader research, aimed at investigating the structure of the mental space of our imagination. It is no surprise that one of Escher's main sources of inspiration is the geometry of the Alhambra arabesques, which can be extended ad infinitum.

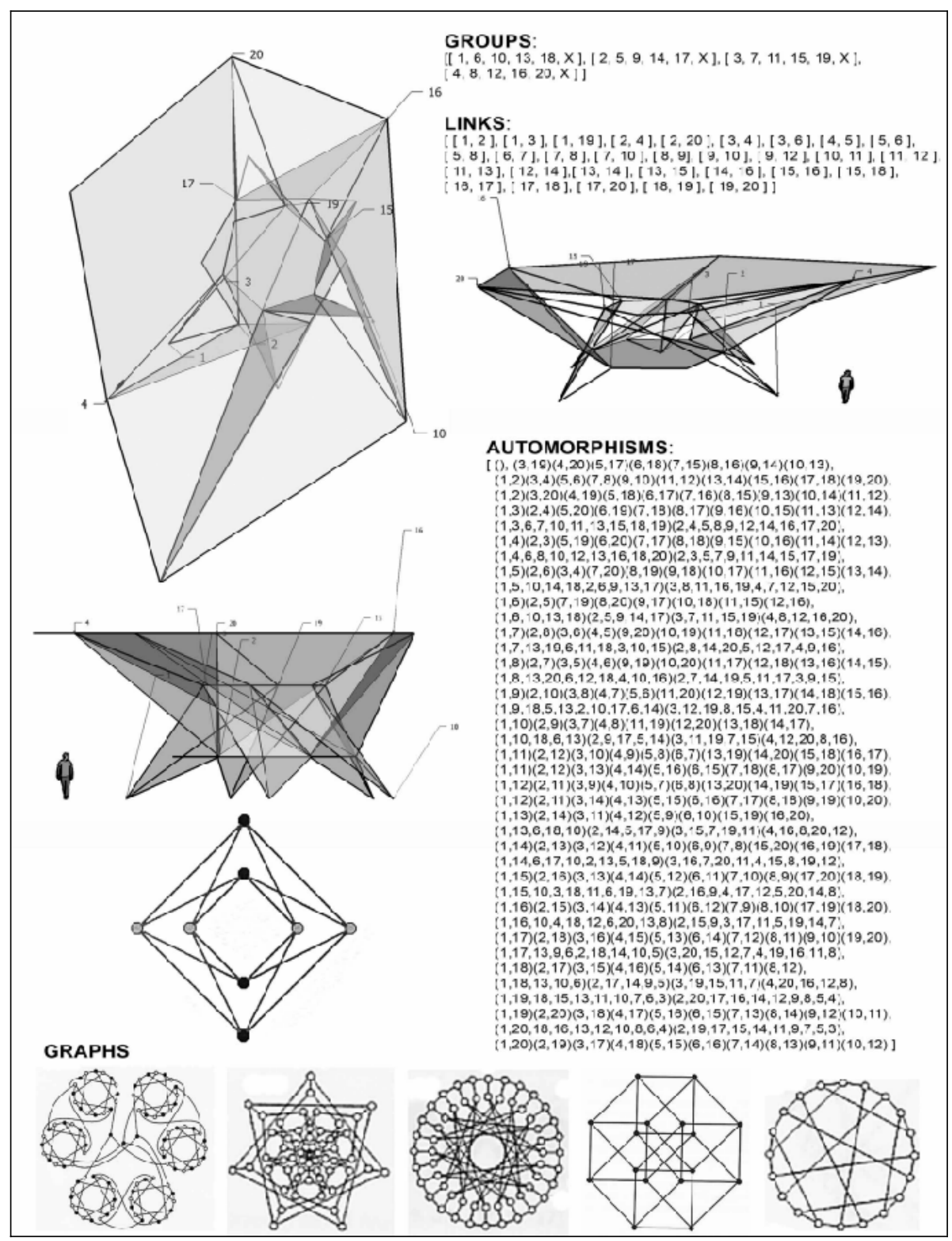

Fig. 2

312 G. FERRERO, C. COTTI, M. Rossi, C. TEDESCHI - Geometries of Imaginary Space 
Recent studies by Peter J. Lu and Paul Steinhardt of Harvard University [Lu and Steinhardt 2007] seem to demonstrate that aperiodic tessellation was already known in ancient times. In the mosaics of the mosque of Darb-i Imam in Iran (1453) never repetitive patterns (girih) are present: they are made from four or five different tiles (decagon, pentagon, rhombus, hexagon, triangle), joined to form decorations that are different from the traditional ones. Their creation relies on the same mathematical principles governing aperiodic Penrose tilings.

Girih and arabesques, kolam and Celtic knots, and rose windows are two-dimensional structures, but they sometimes evoke the planar projection of spatial geometries. Regular patterns similar to these geometrical structures can be found in graph theory and in permutation groups.

\section{Experimenting with modern mathematical models}

The architectural examples mentioned above almost seem to satisfy completely the many opportunities offered by the "classical" (linear, Euclidean and non-Euclidean) geometries, so we tried to make use of other geometries in order to obtain new "exotic", "symmetry effects".

We tried to test the use of some algebraic structures, such as group theory and graph theory, with the aim of building structural and architectural grids.

In order to obtain new types of "symmetries" (i.e., partial automorphisms), we started by constructing (according to the second part of Klein's Erlangen program [Klein 1893]) a geometry with a given group of automorphisms (fig. 2). We are trying to apply this idea to construct regular connected graphs (according the idea of "free mobility" due to Sophus Lie) that can be read as partial plans for a building.

There are a lot of algebraic and combinatorial results of graph and group theory that are suitable for creating and examining such graphs. As a first experiment, for example, we can use one of Frucht's results [Frucht 1949] to obtain, for each abstract group G, a regular graph of degree three having $G$ as a group of automorphisms; this can be useful for easily obtaining (by only fixing a suitable set of points on the ground) many isostatic skeletons. Obviously, by filtering Cayley graphs, we can obtain other types of skeletons that may be interesting: the structure of the involved group $G$ seems to produce useful suggestions for setting in the space the skeleton points, mainly when $\mathrm{G}$ is a product of (or generated by) two, three or four of its subgroups. Maybe we can obtain nice planar regular graphs suitable for building something such as geodetic domes or at least regular balls (with a hard center). Similarly we can try to get Hamiltonian graphs to be transformed in the skeleton of a tensegrity structure taking a cycle as a set of tie-beam.

\section{Conclusion}

Therefore what we may see is how much algebraic studies are involved in development of both non-Euclidean geometries and topological architecture. A continuous fil rouge ties polyhedra, kaleidoscopes, and Fuller's geodesic inventions to the formal research of some modern artists who are inquiring into n-dimensional spaces, such as Attilio Pierelli and Anish Kapoor. 
In addition to his work on jewels and sculptures, Attilio Pierelli conceived a fourdimensional church for Santa Maria del Rosario in Viterbo (Italy) and the model, inspired by the hypercube, has been accepted by the bishop (fig. 3). At this writing, however, it is still in the design phase.

Admiring the Cloud Gate, also known as "the Bean" (2005), at the Millennium Park in Chicago, designed by Anish Kapoor, is a really interesting experience: under "the Bean", there are so many reflections that they can hardly be counted (fig. 4).
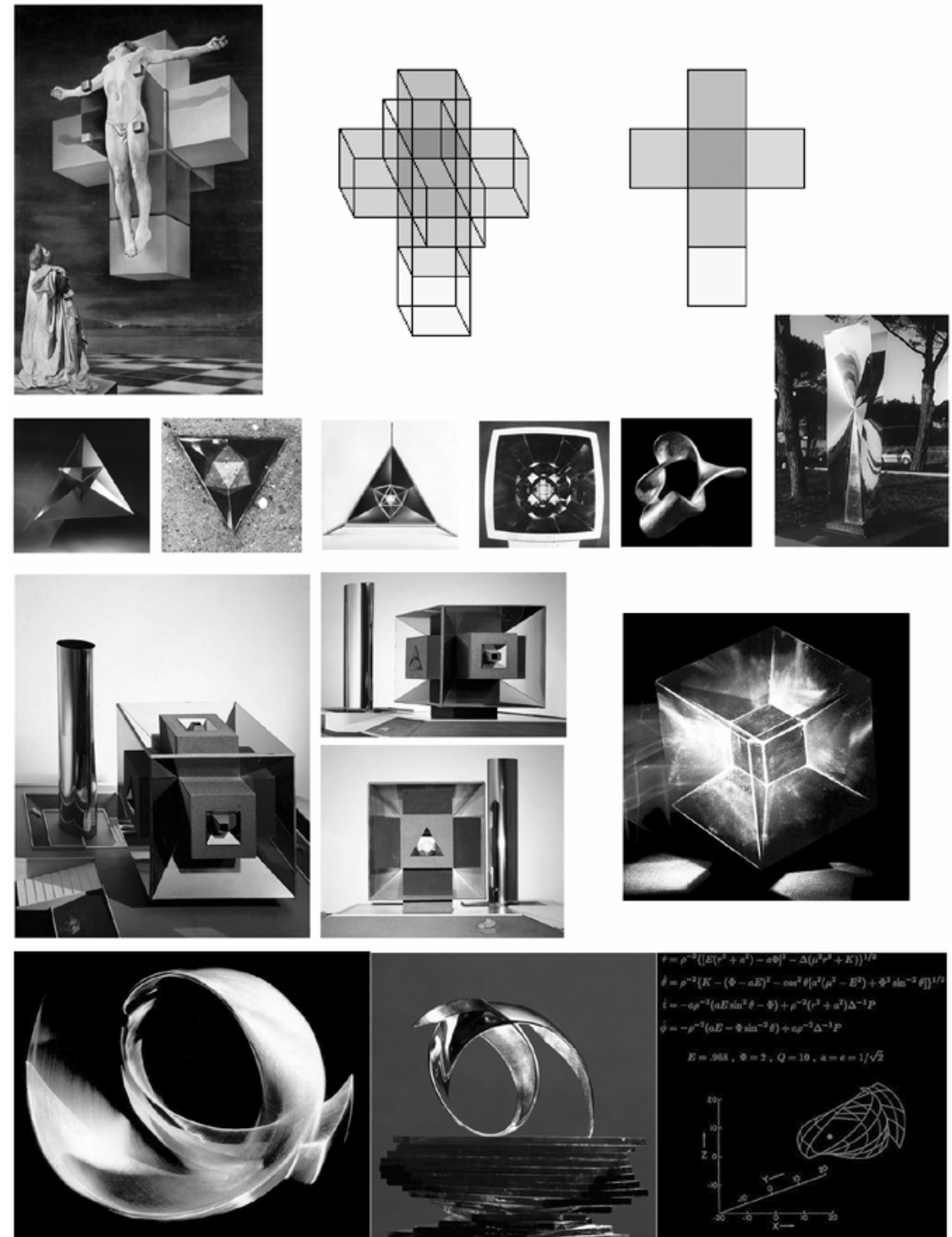

Fig. 3. 

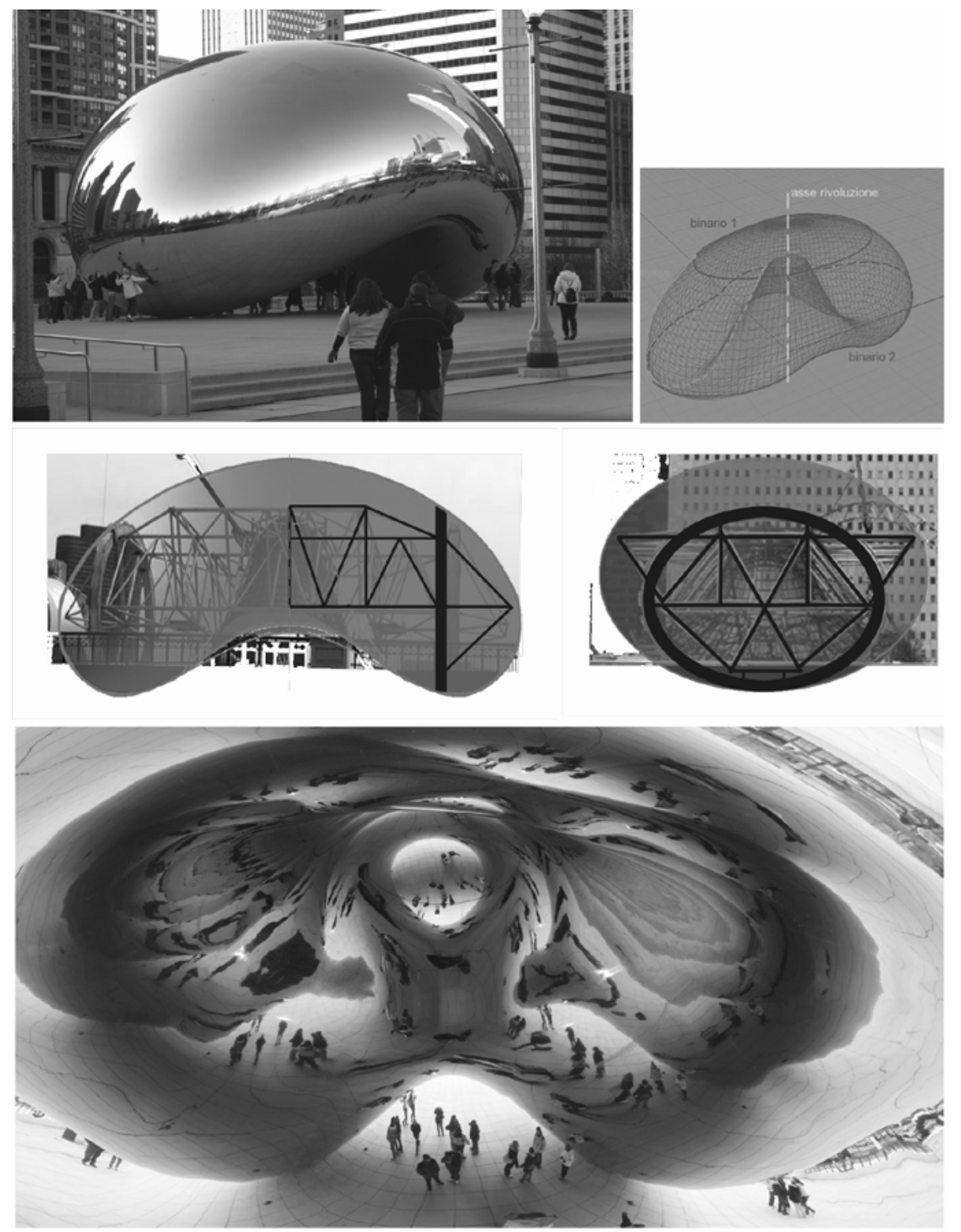

Fig. 4.

Further, the surrounding buildings are mirrored on the inside surface, but everything is deformed and altered, moving and changing with the spectator's own movement; the straight line obviously becomes a curve, but sometimes it changes direction.

It is not easy to replicate the peculiar reflections on the Cloud Gate's continuous surface, not even with a simplified and controlled 3D model. However architecture has never before witnessed anything as close to an $\mathrm{n}$-dimensional space as this. 


\section{Notes}

1. In 1996 Coxeter published a critic of Escher "Circle Limit III" where he demonstrated through trigonometry the mathematical precision of the work.

2. So called in honour of Buckminster Fuller, fullerenes earned their discoverers, Harold Kroto, Robert Curl and Richard Smalley, the 1996 Nobel prize for chemistry. Spherical fullerenes are called buckyballs, while cylindrical ones are called buckytubes.

3. Also called deconstruction, it is a postmodern movement in architecture. Decontructivist architects are F. O. Gehry, D. Libeskind, R. Koolhaas, P. Eisenman, Z. M. Hadid, the group Coop Himmelblau and B. Tschumi.

\section{References}

EATON, L. K. 1998. Fractal Geometry in the Late Work of Frank Lloyd Wright: The Palmer House. Pp. 23-38 in Nexus II: Architecture and Mathematics. Kim Williams, ed. Fucecchio (Florence): Edizioni dell'Erba.

ERnST, Bruno. 1995. The Magic Mirror of M.C. Escher. Benedikt Taschen Verlag.

EsCHER, M.C. 1982. His life and complete graphic work. New York: Abradale Press \& H. M. Abrams.

FRUCHT, R. 1949. Graphs of degree three with a given abstract group. Canadian Journal of Mathematics 1: 365-378.

GOMBRICHT, Ernst. 1979. The Sense of Order. London: Phaidon.

Gorman, Michael J. 2005. Buckminster Fuller Architettura in movimento. Geneva and Milan: Skira.

KLEIN, F. 1893. Vergleichende Betrachtung ueber neue geometriche Forschungen (Erlangen Program). Mathematische Annalen 43: 63-100. Eng. trans. Mellen Haskell, Bulletin of the New York Mathematical Society 2 (1892-1893): 215-249.

Lu, Peter J. and Paul J. STEINHARDT. 2007. Decagonal and Quasi-crystalline Tilings in Medieval Islamic Architecture. Science 315: 1106-1110.

PANOFSKY, Erwin. 1997. Perspective as Symbolic Form. Christopher Wood, trans. New York: Zone Books. (Originally published as "Die Perspektive als «symbolische Form»", Vorträge der Bibliothek Warburg, Leipzig-Berlin: B. G. Teubner, 1927.)

SEMPER, Gottfried. 1960-63. Der Stil. Munich.

\section{About the authors}

Giovanni Ferrero earned his Ph.D in mathematics at the University of Torino. He is full professor in the Department of Mathematics at the University of Parma, where he teaches algebra. He works on near-rings, BIB-designs and STS.

Celestina Cotti earned her Ph.D in mathematics at the University of Parma. She is associate professor at the University of Parma, where she teaches geometry and algebra in the Faculty of Architecture and commutative algebra in the department of mathematics. She works on near-rings.

Michela Rossi received her degree in architecture at the University of Florence and her Ph.D in architectural survey and representation at the University of Palermo in 1993. She is associate professor at the Politecnico di Milano, where she teaches drawing in design courses. Her research interests center on relationships between drawing, geometry and architecture.

Cecilia Tedeschi earned her degree in architecture from the University of Milan in 1995, and her Ph.D in civil engineering from the University of Parma in 2006. Since 1997 she has been involved in the study of drawing, computer graphics and architectural survey of historical buildings and urban environment. 